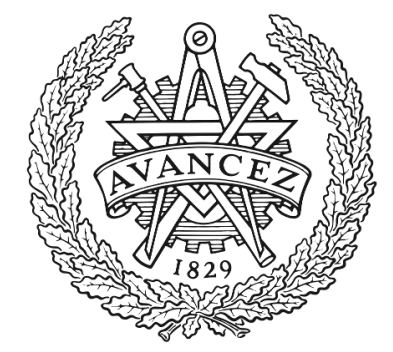

CHALMERS

UNIVERSITY OF TECHNOLOGY

\title{
Investigation of the surface species during temperature dependent dehydrogenation of naphthalene on $\mathrm{Ni}(111)$
}

Downloaded from: https://research.chalmers.se, 2023-04-26 10:22 UTC

Citation for the original published paper (version of record):

Marks, K., Yazdi, M., Piskorz, W. et al (2019). Investigation of the surface species during temperature dependent dehydrogenation of naphthalene on $\mathrm{Ni}(111)$. Journal of Chemical Physics, 150(24). http://dx.doi.org/10.1063/1.5098533

N.B. When citing this work, cite the original published paper. 


\section{Investigation of the surface species during temperature dependent dehydrogenation of naphthalene on $\mathrm{Ni}(111)$}

Cite as: J. Chem. Phys. 150, 244704 (2019); https://doi.org/10.1063/1.5098533

Submitted: 03 April 2019 . Accepted: 27 May 2019 . Published Online: 27 June 2019

Kess Marks (D), Milad Chadami Yazdi, Witold Piskorz (D), Konstantin Simonov, Robert Stefanuik, Daria Sostina, Ambra Guarnaccio (D), Ruslan Ovsyannikov, Erika Giangrisostomi, Yasmine Sassa, Nicolas Bachellier, Matthias Muntwiler (D), Fredrik O. L. Johansson (D), Andreas Lindblad (D), Tony Hansson (D), Andrzej Kotarba (D), Klas Engvall, Mats Göthelid (D), Dan J. Harding (D), and Henrik Öström (D)

\section{COLLECTIONS}

EP

This paper was selected as an Editor's Pick
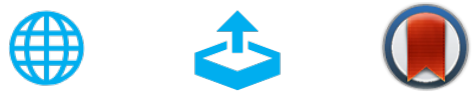

\section{ARTICLES YOU MAY BE INTERESTED IN}

Vibrational spectra of dissociatively adsorbed $\mathrm{D}_{2} \mathrm{O}$ on $\mathrm{Al}$-terminated $\alpha-\mathrm{Al}_{2} \mathrm{O}_{3}(\mathrm{OOO} 1)$ surfaces from ab initio molecular dynamics

The Journal of Chemical Physics 150, 244701 (2019); https://doi.org/10.1063/1.5099895

Glass polymorphism in TIP4P/2005 water: A description based on the potential energy landscape formalism

The Journal of Chemical Physics 150, 244506 (2019); https://doi.org/10.1063/1.5100346

Vibronic coupling models for donor-acceptor aggregates using an effective-mode scheme: Application to mixed Frenkel and charge-transfer excitons in oligothiophene aggregates The Journal of Chemical Physics 150, 244114 (2019); https://doi.org/10.1063/1.5100529

The Journal

of Chemical Physics

The Emerging Investigators Special Collection and Awards Recognizing the excellent work of early career researchers! 


\title{
Investigation of the surface species during temperature dependent dehydrogenation of naphthalene on $\mathrm{Ni}(111)$
}

Cite as: J. Chem. Phys. 150, 244704 (2019); doi: 10.1063/1.5098533

Submitted: 3 April 2019 - Accepted: 27 May 2019 •

Published Online: 27 June 2019

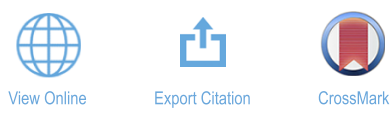

\begin{abstract}
Kess Marks, ${ }^{1}$ (D) Milad Ghadami Yazdi, ${ }^{2}$ Witold Piskorz, ${ }^{3}$ (D) Konstantin Simonov, ${ }^{4}$ Robert Stefanuik, Daria Sostina, ${ }^{5}$ Ambra Guarnaccio, ${ }^{6}$ (i) Ruslan Ovsyannikov, ${ }^{7}$ Erika Giangrisostomi, ${ }^{7}$ Yasmine Sassa, Nicolas Bachellier, ${ }^{5}$ Matthias Muntwiler, ${ }^{5}$ (D) Fredrik O. L. Johansson, ${ }^{4}$ (D) Andreas Lindblad, ${ }^{4}$

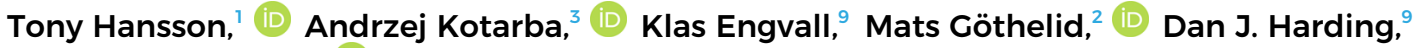
and Henrik Öström?

\author{
AFFILIATIONS \\ ${ }^{1}$ Department of Physics, Fysikum, Stockholm University, 10691 Stockholm, Sweden \\ ${ }^{2} \mathrm{SCl}$, Material and Nanophysics, KTH Royal Institute of Technology, 16440 Kista, Sweden \\ ${ }^{3}$ Faculty of Chemistry, Jagiellonian University in Kraków, Gronostajowa 2, 31-387 Kraków, Poland \\ 4Department of Physics and Astronomy, Uppsala University, 75120 Uppsala, Sweden \\ ${ }^{5}$ Paul Scherrer Institut, 5232 Villigen PSI, Switzerland \\ ${ }^{6}$ CNR-ISM-Institute of Structure of Matter —Tito Scalo Unit, C/da S. Loja, 85050 Tito Scalo, Potenza, Italy \\ ${ }^{7}$ Institute for Methods and Instrumentation in Synchrotron Radiation Research FG-ISRR, \\ Helmholtz-Zentrum Berlin für Materialien und Energie, 12489 Berlin, Germany \\ ${ }^{8}$ Department of Physics, Chalmers University of Technology, SE-412 96 Göteborg, Sweden \\ ${ }^{9}$ Department of Chemical Engineering, KTH Royal Institute of Technology, 10044 Stockholm, Sweden
}

\begin{abstract}
The temperature dependent dehydrogenation of naphthalene on $\mathrm{Ni}(111)$ has been investigated using vibrational sum-frequency generation spectroscopy, X-ray photoelectron spectroscopy, scanning tunneling microscopy, and density functional theory with the aim of discerning the reaction mechanism and the intermediates on the surface. At $110 \mathrm{~K}$, multiple layers of naphthalene adsorb on $\mathrm{Ni}(111)$; the first layer is a flat lying chemisorbed monolayer, whereas the next layer(s) consist of physisorbed naphthalene. The aromaticity of the carbon rings in the first layer is reduced due to bonding to the surface Ni-atoms. Heating at $200 \mathrm{~K}$ causes desorption of the multilayers. At $360 \mathrm{~K}$, the chemisorbed naphthalene monolayer starts dehydrogenating and the geometry of the molecules changes as the dehydrogenated carbon atoms coordinate to the nickel surface; thus, the molecule tilts with respect to the surface, recovering some of its original aromaticity. This effect peaks at $400 \mathrm{~K}$ and coincides with hydrogen desorption. Increasing the temperature leads to further dehydrogenation and production of $\mathrm{H}_{2}$ gas, as well as the formation of carbidic and graphitic surface carbon.
\end{abstract}

Published under license by AIP Publishing. https://doi.org/10.1063/1.5098533

\section{INTRODUCTION}

In the process of biomass gasification for production of renewable energy, unwanted by-products, heavier hydrocarbons, and polyaromatic hydrocarbons (PAHs), referred to as tars, prevent complete utilization of the gas-product and up-scaling of the production method. ${ }^{1-3}$ Therefore, an exploration of methods to reduce the negative effects of the tar by-product is required. Catalytic reforming is regarded as a promising method for removal of tar by converting it into useful permanent gases such as $\mathrm{CO}$ and $\mathrm{H}_{2}$. Nickelbased catalysts are commonly used in catalytic steam reforming and are also considered to be a viable and economical option for 
biomass gasification. ${ }^{4,5}$ Although nickel is an effective catalyst for hydrocarbon dehydrogenation, it also catalyzes graphite formation, which leads to catalyst poisoning. ${ }^{6}$ Naphthalene is a major component in most tar and has been used as a model molecule for catalytic studies. It is also an intermediate in the decomposition of other PAHs, which makes decomposition of naphthalene an important basis for the understanding of the complete steam reforming of tar.

In a previous structural investigation of naphthalene on $\mathrm{Ni}(111){ }^{7}$ we have shown that naphthalene molecules adsorb flat on smooth terraces, preferring a geometry of aromatic rings positioned over Ni-bridge sites. Temperature programmed desorption (TPD) showed that desorption of $\mathrm{H}_{2}$ occurs in two steps, where the second step coincides with the conversion of carbon atoms to a graphenelike structure, causing rapid passivation of the catalyst. ${ }^{7}$ In the present study, we aim to elucidate the mechanism of naphthalene dehydrogenation and graphene formation on the $\mathrm{Ni}(111)$ surface by investigating the surface species during the temperature dependent dehydrogenation.

Adsorption and reaction studies of naphthalene on nickel are not abundant in the literature, but the adsorption of naphthalene on other metals such as $\mathrm{Pt}(111),{ }^{8-14} \mathrm{Cu}(111),{ }^{15-18} \mathrm{Rh}(111),{ }^{14,19}$ and $\operatorname{Ag}(111)^{20-23}$ has been investigated both experimentally and theoretically. Dahlgren and Hemminger ${ }^{9}$ studied naphthalene on $\operatorname{Pt}(111)$ using low-energy electron diffraction (LEED) and showed that at room temperature the ordering of naphthalene molecules is randomized, but that mild heating leads to an ordered $(6 \times 3)$ overlayer. Santarossa et al. ${ }^{14}$ used density functional theory (DFT) calculations to show that a flat lying di-bridge-7 is the optimal adsorption configuration of naphthalene on Pt, Pd, and Rh. Similar findings were recently reported by Kolsbjerg et al. ${ }^{24}$ for naphthalene on $\mathrm{Pt}(111)$ and by Yazdi et al. ${ }^{7}$ for naphthalene on $\mathrm{Ni}(111)$ where the di-bridge-7 is the most favored configuration. Additionally, DFT calculations by Yazdi et al. show that the adsorption of naphthalene is accompanied by a pronounced de-aromatization, similar to that seen for benzene on $\mathrm{Ni}(100)$ and $\mathrm{Cu}(110)$.

The dehydrogenation of naphthalene has been studied by both Lin et al. ${ }^{19}$ and Dahlgren and Hemminger. ${ }^{8}$ Lin et al. used LEED, Auger electron spectroscopy (AES), and TPD to show that naphthalene on $\mathrm{Rh}(111)$ transforms from a $(3 \sqrt{3} \times 3 \sqrt{3}) \mathrm{R} 30^{\circ}$ structure to a $(3 \times 3)$ structure in the temperature range of $398-423 \mathrm{~K}$ and to a disordered layer at $448 \mathrm{~K}$. They found the first $\mathrm{H}_{2}$ desorption peak at 578 K. Dahlgren and Hemminger ${ }^{8}$ studied naphthalene on $\mathrm{Pt}(111)$ using LEED, AES, and TPD and found that, at near saturation coverage, naphthalene forms an ordered layer which is stable until the onset of hydrogen desorption at $470 \mathrm{~K}$. After the desorption process, the $\mathrm{Pt}(111)$ surface is covered with carbon residue. In both cases, the only desorption process observed is the desorption of molecular hydrogen accompanied by irreversible structural changes in the naphthalene overlayer. This is consistent with the findings of Yazdi et al. ${ }^{7}$ where scanning tunneling microscopy (STM) and TPD were used to study naphthalene on $\mathrm{Ni}(111) . \mathrm{H}_{2}$ desorption coincides with the disappearance of ordered naphthalene patches in the temperature range of $380-600 \mathrm{~K}$ with the first desorption peak at $450 \mathrm{~K}$. At temperatures above $580 \mathrm{~K}$, carbon chain and network growth is reported, and it is assumed that a graphenelike layer is formed. Graphene growth from precursor PAHs on $\mathrm{Ni}(111)$ has been identified in several investigations, ${ }^{26-28}$ and growth mechanisms, either via traditional nucleation ${ }^{26,29}$ or via a Ni-carbide intermediate, ${ }^{30-32}$ have been suggested.

Here, we present a combination of vibrational sum-frequency generation spectroscopy (SFG), X-ray photoelectron spectroscopy (XPS), and STM measurements and DFT calculations, in order to understand the mechanism of adsorption and temperature driven dehydrogenation of naphthalene on $\mathrm{Ni}(111)$ in Ultra-High Vacuum (UHV). We show that partial dehydrogenation of the naphthalene molecules is accompanied by a change of the geometry of the molecules as the dehydrogenated carbons coordinate more strongly to the nickel surface atoms. As a result, the naphthalene molecules tilt with respect to the surface and the carbon atoms on the other side of the molecule move away from the surface retrieving some $\mathrm{sp}^{2}$ characteristics and some of the aromaticity that was lost upon adsorption. Further heating continues the dehydrogenation, leading to the desorption of $\mathrm{H}_{2}$ and the formation of a mixture of carbidic and graphitic surface carbon.

\section{EXPERIMENTAL DETAILS}

Pure naphthalene (99\%) was purchased from Sigma-Aldrich and dosed through a precision leak valve. Doses are given in $\mathrm{L}$ (Langmuir), where $1 \mathrm{~L}=1 \times 10^{-6}$ Torr s. It takes about $3.5 \mathrm{~L}$ to form a saturated monolayer at room temperature. Additional dosing at room temperature does not increase the surface coverage. The $\mathrm{Ni}(111)$ crystal was purchased from the Surface Preparation Laboratory (SPL) in the Netherlands and was polished and aligned to within less than $0.1^{\circ}$ from the (111) plane.

Sum-frequency generation spectroscopy was performed in an UHV chamber with a base pressure of $1 \times 10^{-10}$ Torr. The system is equipped with a quadrupole mass spectrometer (QMS), LEED optics, and an ion gun used for sample cleaning. A thermocouple was spot-welded on the $\mathrm{Ni}(111)$ sample for temperature monitoring. The sample temperature was controlled through a combination of liquid nitrogen cooling and resistive and/or electron bombardment heating. The sample was cleaned through cycles of $\mathrm{Ar}^{+}$-sputtering at room temperature and annealing up to $1100 \mathrm{~K}$. The quality of the surface was monitored using LEED. Naphthalene layers were prepared by dosing $5 \mathrm{~L}$ of naphthalene at $110 \mathrm{~K}$ which results in a saturated monolayer and at least one additional layer.

A commercial Ti:sapphire amplifier system which produces $800 \mathrm{~nm}$ pulses with a $\leq 50 \mathrm{fs}$ pulse duration and a power of $3.7 \mathrm{~W}$ at a repetition rate of $1 \mathrm{kHz}$ was used for the SFG experiments. A tunable optical parametric amplifier (TOPAS) and a noncollinear difference frequency generator (n-DFG) were used to convert the $800 \mathrm{~nm}$ light to wavelengths in the mid-infrared (IR) region (tunable within $2.6-11 \mu \mathrm{m}$ ). For these experiments, the mid-IR was set to around $3500 \mathrm{~nm}$ to cover the naphthalene $\mathrm{C}-\mathrm{H}$ stretching region. Part of the $800 \mathrm{~nm}$ light was spectrally narrowed to a bandwidth of $12 \mathrm{~cm}^{-1}$ using a pulse shaper and overlapped on the sample in space and time with the mid-IR light. The two laser beams were sent into the UHV chamber through an IR-transparent $\mathrm{BaF}_{2}$ window. After interaction with the surface, the $800 \mathrm{~nm}$ light was filtered using a $750 \mathrm{~nm}$ short-pass edge filter so that only the generated SFG light was sent into the spectrometer. An intensified charge-coupled device (ICCD) camera was used to record the SFG spectra with a final resolution of $14 \mathrm{~cm}^{-1}$. The SFG data were fit by assuming that the second order susceptibility $\chi^{(2)}$ can be written as 


$$
\chi^{(2)}=\chi_{N R}^{(2)}+\chi_{R}^{(2)}=\chi_{N R}^{(2)}+\sum_{n} \frac{A_{n} e^{i \varphi_{n}}}{\left(\omega-\omega_{n}\right)+i \Gamma_{n}},
$$

where $\chi^{(2)} \mathrm{NR}$ is the nonresonant contribution as measured from a clean surface and $\chi^{(2)}{ }_{R}$ is the resonant contribution, with $A_{n}, \varphi_{n}, \omega_{n}$, and $\Gamma_{\mathrm{n}}$ being the amplitude, phase, frequency, and half-width of the resonance, respectively.

X-ray photoelectron spectroscopy measurements were performed at the LowDosePES ${ }^{34}$ end-station of the PM4 beamline at the synchrotron Berlin electron storage ring society for synchrotron radiation (BESSY) II at Helmholtz-Zentrum Berlin. The main analysis chamber had a base pressure of $1 \times 10^{-10}$ Torr and is equipped with a VG Scienta ARTOF-2 electron energy analyzer. The sample preparation was done in a separate chamber equipped with an ion-gun, leak valves, and resistive heating up to $900 \mathrm{~K}$. The XPS measurements were performed at room temperature at $380 \mathrm{eV}$ photon energy.

Low temperature STM (Omicron) was performed at the Photoemission and Atomic Resolution Laboratory (PEARL) at Swiss Light Source (SLS), Switzerland. The sample was cleaned according to the procedure described earlier. Sample quality and surface order were checked with LEED. Naphthalene was dosed at room temperature into the preparation chamber through a precision leak valve. Since it was necessary not to saturate the surface in order to measure the height difference between adsorbates and the surface, only $1 \mathrm{~L}$ of naphthalene was dosed. After dosing, the sample was moved to the STM chamber for imaging at $4 \mathrm{~K}$, using etched gold tips. The tunnel bias was applied to the sample.

\section{COMPUTATIONAL DETAILS}

The adsorption structure of naphthalene on $\mathrm{Ni}(111)$ was optimized using the DFT PBE $+\mathrm{D} 2^{35}$ level of theory implemented in the VASP code. ${ }^{36,37}$ The energy cutoff was $600 \mathrm{eV}$, and the IBZ sampling was done at the $\Gamma$ point because of the large size of the unit cell. Tight convergence criteria $\left(10^{-7} \mathrm{eV}\right.$ for the electronic structure optimization and $10^{-5} \mathrm{eV} / \AA$ for ionic relaxation) were used. The cell parameters of the bulk phase were calculated as a minimum of the Birch-Murnaghan equation of state ${ }^{38}$ fitting. As a convergence accelerator, Methfessel-Paxton ${ }^{39}$ smearing was used with the width parameter $\sigma=0.1 \mathrm{eV}$. The bond orders (BO) were calculated via the DDEC6 method. ${ }^{40,41}$ The surface model was a Ni(111) rhombohedral slab of $19.93 \times 19.93 \times 21.10 \AA^{3}$ unit cell size $(8 \times 8 \times 4$ $\mathrm{Ni}$ atoms with 2 bottom layers fixed during ionic relaxation). We computed a spin-polarization of 0.64 bohr magneton for the Ni slab, which is close to the literature value of $0.61 .^{42}$ The vacuum layer was $15.14 \AA$ thick. The entropic contributions to the free energy and the SFG active modes were calculated using the classical harmonic vibrational analysis. The energy barriers were calculated with the use of the nudged elastic band (NEB) methodology (5 optimized images), using the VTST package.

\section{RESULTS AND DISCUSSION}

\section{SFG measurements}

Figure 1 shows the vibrational SFG spectra for naphthalene on $\mathrm{Ni}(111)$ in the $\mathrm{C}-\mathrm{H}$ stretching region. The bottom spectrum shows

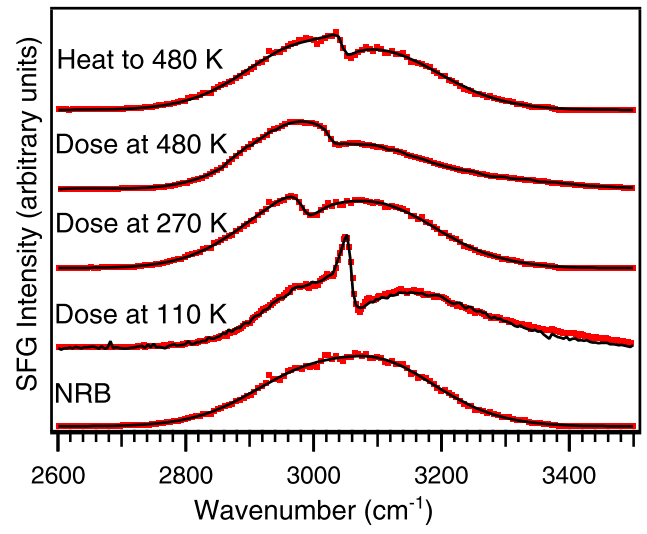

FIG. 1. Normalized SFG spectra (red squares) and corresponding fits (black lines) recorded for naphthalene adsorbed on $\mathrm{Ni}(111)$ dosed at 110,270 , and $480 \mathrm{~K}$. Fits place the resonances at 3057,2985 , and $3029 \mathrm{~cm}^{-1}$, respectively. The bottom spectrum shows the nonresonant background (NRB) recorded for a clean Ni(111) sample. The top spectrum shows the SFG spectrum for naphthalene dosed at 270 $\mathrm{K}$ and flashed to $480 \mathrm{~K}$ afterwards; the fit places the resonance at $3057 \mathrm{~cm}^{-1}$. All spectra were recorded at $270 \mathrm{~K}$ except for the low-temperature spectrum which was both dosed and recorded at $110 \mathrm{~K}$.

the nonresonant background (NRB), recorded from a clean $\mathrm{Ni}(111)$ surface before adsorption of naphthalene, and follows the spectral profile of the broadband infrared pulse. The red squares represent the measurement, and the black solid lines are fits to the data. Sumfrequency generation spectroscopy is subject to stricter selection rules than both Raman and infrared absorption spectroscopy. Essentially, only vibrations that are both Raman and infrared active will be active in SFG. ${ }^{44}$ Additionally, the metal surface selection rule states that only vibrations with a dynamic dipole moment along the surface normal will be active. ${ }^{45}$

A $5 \mathrm{~L}$ dose at $110 \mathrm{~K}$ results in a chemisorbed monolayer and a physisorbed multilayer of naphthalene. In the spectrum recorded after dosing at $110 \mathrm{~K}$, one sharp resonance is observed at $3057 \mathrm{~cm}^{-1}$, corresponding to multilayer naphthalene, in addition to the nonresonant background. The observed resonant frequency is in the middle of the aromatic $\mathrm{C}-\mathrm{H}$ stretching region and matches the frequencies found by Jakob and Menzel ${ }^{46}$ and Lehwald et al. ${ }^{47}$ for multilayer benzene on $\mathrm{Ru}(001)$ and $\mathrm{Ni}(111)$, respectively. Dosing at $270 \mathrm{~K}$ should result in a monolayer of naphthalene since this temperature is above the desorption temperature of the naphthalene multilayers. The SFG spectrum of this preparation shows a resonance at $2985 \mathrm{~cm}^{-1}$, which is on the low-frequency side of the multilayer peak and on the edge of the aliphatic $\mathrm{C}-\mathrm{H}$ stretching region. This resonance can indeed be attributed to the chemisorbed monolayer naphthalene in which we expect a loss of aromaticity and more $\mathrm{sp}^{3}$ characteristics, similar to adsorption of other unsaturated hydrocarbons on metal surfaces. ${ }^{48,49}$ Thomas et al. ${ }^{49}$ observed similar $\mathrm{sp}^{3}$ $\mathrm{C}-\mathrm{H}$ stretches at $2980 \mathrm{~cm}^{-1}$, attributed to dienyl moieties formed by coordination of the carbon atoms to the metal, using vibrational electron energy loss spectroscopy (HREELS) for benzene on $\operatorname{Pt}(110)$.

Dosing at $270 \mathrm{~K}$ to form a monolayer of naphthalene and subsequent flashing to $480 \mathrm{~K}$ to start dehydrogenation results in a 
spectrum with a resonance at $3057 \mathrm{~cm}^{-1}$, while dosing directly at $480 \mathrm{~K}$ gives a resonance at $3029 \mathrm{~cm}^{-1}$. The difference in vibrational frequency between the spectra of the two preparation procedures is attributed to the fact that, when dosing at $480 \mathrm{~K}$, the naphthalene is thermally activated while adsorbing and already dehydrogenating $^{7,8,19}$ [see Fig. 2(c)]. This can affect the adsorption and coverage of the molecules on the surface and thus the vibrational frequencies. In order to gain further understanding of these species, we performed temperature programmed SFG measurements.

Figure 2(a) shows a false color plot of a temperature dependent SFG measurement of $10 \mathrm{~L}$ of naphthalene on $\mathrm{Ni}(111)$ dosed at $110 \mathrm{~K}$. Each horizontal line represents one recorded spectrum. Fits of individual slices reveal the presence of two resonances, one at $3057 \mathrm{~cm}^{-1}$ and one at $3003 \mathrm{~cm}^{-1}$. Figure 2(d) depicts four horizontal slices taken from Fig. 2(a) at 150, 300, 450, and $600 \mathrm{~K}$ to better illustrate the observed resonances. The resonance at $3057 \mathrm{~cm}^{-1}$ is the same as that found for dosing at $110 \mathrm{~K}$; the second resonance is shifted with respect to the frequency observed in the previous section $\left(2985 \mathrm{~cm}^{-1}\right)$ due to the lower dosing temperature used for this experiment which results in a slightly different adsorbate layer. The data were then fit as a function of temperature assuming the presence of these two resonances and using the same fitting parameters for each slice, only allowing the amplitude to vary freely in the fits. The fitted amplitudes for both resonances are plotted as a function of temperature in Fig. 2(b), showing the evolution of the two resonances. Figure 2(c) depicts a reproduction of TPD results ${ }^{7}$ of the same system, recorded with a ramping rate of $50 \mathrm{~K} / \mathrm{min}$.

At temperatures below $200 \mathrm{~K}$, we again observe a resonance at $3057 \mathrm{~cm}^{-1}$, originating from the aromatic $\mathrm{C}-\mathrm{H}$ stretch vibrations of the physisorbed multilayers of naphthalene. Around $200 \mathrm{~K}$, this resonance disappears as the multilayer desorbs, consistent with the TPD measurements. The $3003 \mathrm{~cm}^{-1}$ resonance that appears originates from the flat monolayer naphthalene with increased $\mathrm{sp}^{3}$ characteristics of the carbon atoms. This resonance grows at temperatures above $200 \mathrm{~K}$, as the vibrations of the monolayer are no longer suppressed by the multilayers on top. Between $200 \mathrm{~K}$ and $360 \mathrm{~K}$, the $3003 \mathrm{~cm}^{-1}$ feature loses some intensity but is still clearly present, as can be seen in Figs. 2(a) and 2(b). Around $360 \mathrm{~K}$, it decreases more rapidly and simultaneously a new resonance appears, close to the original multilayer peak at $3057 \mathrm{~cm}^{-1}$. Since $360 \mathrm{~K}$ is well above the desorption temperature for physisorbed naphthalene, this resonance must be due to a recovery of $\mathrm{sp}^{2}$ characteristics by the monolayer species. This suggests that some carbon atoms lose contact with the metal as the result of a geometrical change of the adsorbed species upon partial dehydrogenation. This feature also appears in the same temperature region as the onset of hydrogen production detected by earlier TPD studies. This spectral behavior can be expected if the molecule tilts with respect to the surface so that the bond between the nickel surface and the raised carbon atoms lengthens and decreases in strength allowing those carbon atoms to regain some $\mathrm{sp}^{2}$ characteristics, i.e., the molecule goes towards a standing geometry. The signal from this "tilted" molecule at $3057 \mathrm{~cm}^{-1}$ peaks around $400 \mathrm{~K}$ [Fig. 2(b)] and disappears at $500 \mathrm{~K}$; the $3003 \mathrm{~cm}^{-1}$ feature lingers up to $480 \mathrm{~K}$, after which the spectrum resembles the nonresonant background. $\mathrm{H}_{2}$ desorption, however, is known to continue up $650 \mathrm{~K}^{7}$, which implies that although the SFG signal disappears, there are still $\mathrm{C}_{\mathrm{x}}-\mathrm{H}_{\mathrm{y}}$ fragments on the surface that continue to dehydrogenate. These fragments are invisible to SFG which can be rationalized by the sensitivity of this technique for parameters such as surface order, cross sections, and the direction of dynamic dipoles.

Based on the temperature dependence of the tilted species shown in Fig. 2(b), a rough estimate of the energy barrier for the formation of this species can be made. For an effective first order Arrhenius type reaction, the energy barrier would be $27 \pm 2 \mathrm{kcal} / \mathrm{mol}$, based on the fact that the rising slope of the $3057 \mathrm{~cm}^{-1}$ feature is centered around $380 \mathrm{~K}$ and assuming a typical pre-exponential of $10^{13 \pm 1} \mathrm{~s}^{-1}$.

\section{XPS C 1s measurements}

Figure 3 shows the C 1s XPS spectra of naphthalene on Ni(111) dosed and recorded at room temperature after flashing to increasingly high temperatures (300-900 K).

The data were fit using gaussians to obtain the position of the two distinguishable features and the total peak area. The first spectrum, for $300 \mathrm{~K}$, shows one broad feature at $284.6 \mathrm{eV}$, which covers the contributions from the three distinct carbon atoms in the naphthalene molecule. Individual contributions from the different carbon

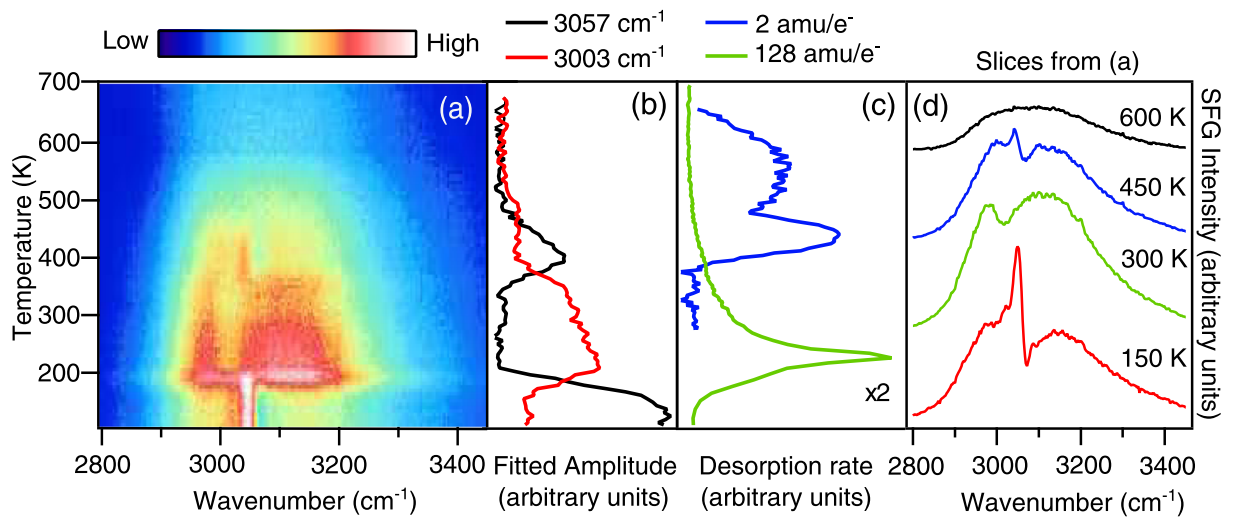

FIG. 2. (a) False color plot showing the temperature dependence of SFG spectra of naphthalene on $\mathrm{Ni}(111)$, recorded with a heating rate of $3 \mathrm{~K} / \mathrm{min}$. Dark blue regions indicate low intensities and white regions indicate high intensities. (b) Temperature dependence of the fitted amplitudes for the resonances at $3057 \mathrm{~cm}^{-1}$ (black) and $3003 \mathrm{~cm}^{-1}$ (red). (c) TPD of naphthalene on $\mathrm{Ni}(111)$ for naphthalene desorption (128 amu/e) and hydrogen desorption (2 amu/e), at a heating rate of $50 \mathrm{~K} / \mathrm{min}$. (d) Selected spectrum slices from (a) at temperatures of 150 , 300,450 , and $600 \mathrm{~K}$. 


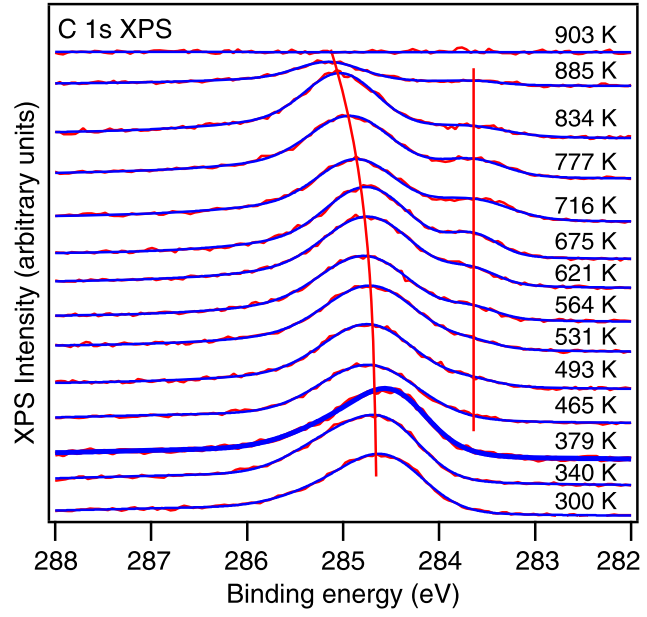

FIG. 3. Carbon 1 s XPS spectra for $5 \mathrm{~L}$ of naphthalene on $\mathrm{Ni}(111)$ dosed at room temperature and flashed to increasingly high temperatures. The recorded spectrum is depicted in red and the corresponding fit in blue. Two vertical red lines indicate the fitted positions of the two main features. The spectrum at $379 \mathrm{~K}$ is indicated in bold; its shape and position are distinguishably different from the other spectra. The naphthalene $\mathrm{C} 1 \mathrm{~s}$ at $300 \mathrm{~K}$ has a feature at $284.6 \mathrm{eV}$ which shows a substantial shift to higher binding energy after $700 \mathrm{~K}$, reaching $285.1 \mathrm{eV}$ at $885 \mathrm{~K}$. Around $465 \mathrm{~K}$, a second feature appears at $283.6 \mathrm{eV}$. Both features have completely disappeared after $900 \mathrm{~K}$.

atoms could not be resolved. The width [full width at half maximum (FWHM)] of this feature becomes narrower with increasing temperatures from $1.1 \mathrm{eV}$ at $300 \mathrm{~K}$ to $0.8 \mathrm{eV}$ at $834 \mathrm{~K}$. This is due to a decrease in the broadening contribution of $\mathrm{C}-\mathrm{H}$ vibrations ${ }^{50,51}$ as $\mathrm{C}-\mathrm{H}$ bonds are broken with increasing temperature.

The spectrum after heating to $379 \mathrm{~K}$ is indicated in bold. Its shape and peak position are distinguishably different from the spectra for lower and higher temperatures. This is consistent with the SFG spectra, where we also observe large changes around this temperature. The spectrum exhibits a shift to slightly lower binding energy, implying an increase in the $\mathrm{sp}^{2}$ characteristics of the carbon atoms, ${ }^{52}$ which would support our interpretation of the SFG measurements. Unfortunately, the temperature resolution of the XPS data does not allow for any further conclusions with regard to the geometry of the molecule as dehydrogenation progresses.

At $465 \mathrm{~K}$, a second distinguishable feature appears at $283.6 \mathrm{eV}$, indicating the formation of carbidic carbon, ${ }^{53,54}$ recently identified as a precursor for graphene growth by Rameshan et al. ${ }^{31}$ The main peak, which at $300 \mathrm{~K}$ is positioned at $284.6 \mathrm{eV}$, shifts to higher binding energy with increasing temperature and shows a substantial shift above $700 \mathrm{~K}$ towards $285.1 \mathrm{eV}$. This can be attributed to the formation of disordered graphitic carbon. ${ }^{52,53}$ All features are gone above $900 \mathrm{~K}$, likely due to migration of the carbon atoms into the bulk of the crystal, as was seen for carbon thin films at similar temperatures by Wiltner and Linsmeier.

\section{STM height distribution measurements}

Scanning tunneling microscopy was used to measure the molecular height distribution after room temperature dosing $1 \mathrm{~L}$ of naphthalene on a clean $\mathrm{Ni}(111)$ surface both at room temperature and heated to $360 \mathrm{~K}$, which is the onset of dehydrogenation. After preparation, the sample was transferred to the STM chamber and cooled down to $4 \mathrm{~K}$ before imaging. The procedure was to prepare the surface, image with the STM, then move the sample for heating, and move back to the STM for imaging of the second preparation. There is no guarantee that the same surface area is imaged; however, the surface was imaged at several spots for each preparation and the images presented are representative of the surface. Figures 4(a) and 4(b) show images of the surface after preparation at room temperature and after heating to $360 \mathrm{~K}$, respectively. The naphthalene molecules have elongated features in Fig. 4(a), a shape consistent with the flat adsorption geometry that is suggested for naphthalene on $\mathrm{Ni}(111)$. ${ }^{7}$ After heating to $360 \mathrm{~K}$, a more irregular surface with larger variations in height is observed. The dark areas in both images can tentatively be assigned to atomic hydrogen binding to the $\mathrm{Ni}(111)$ surface. Although the images are recorded for the same preparation, the coverage is different: 200 molecules/625 $\mathrm{nm}^{2}$ in Fig. 4(a) and 300 molecules $/ 625 \mathrm{~nm}^{2}$ in Fig. 4(b).

Figure 4(c) shows a height histogram of both images obtained by marking and eliminating the background. The blue curve displays the height distribution of the naphthalene molecules after room temperature adsorption. The distribution has a width (FWHM) of $0.2 \AA$ and peaks at $0.4 \AA$ above the nickel surface. This implies that at room temperature practically all molecules lie flat on the surface, in agreement with previous findings. ${ }^{7}$ The red curve in Fig. 4(c) shows the height distribution after heating to $360 \mathrm{~K}$, which is just at the onset of dehydrogenation. The distribution is broader (FWHM $0.3 \AA$ ) with the center shifted to $0.6 \AA$. This means that, at the onset of

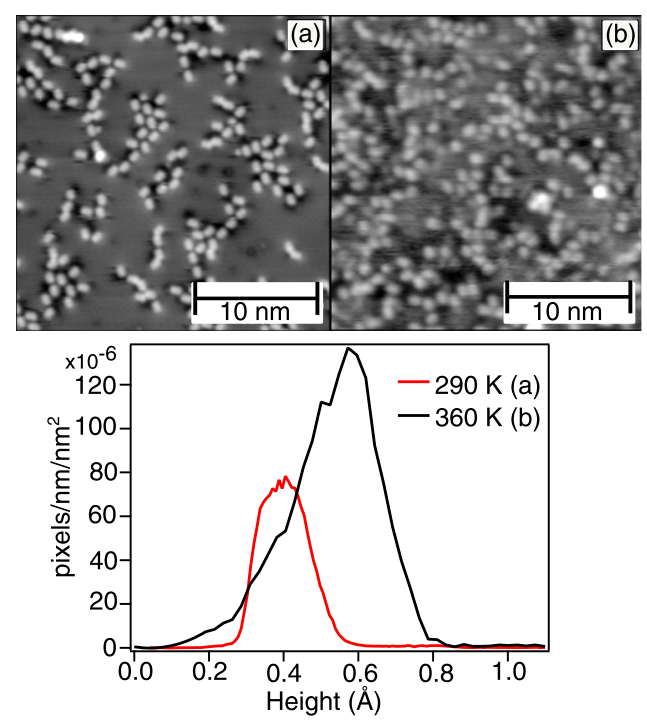

FIG. 4. (a) STM image of naphthalene molecules dosed at room temperature on $\mathrm{Ni}(111)$ captured at $4 \mathrm{~K}(25 \times 25 \mathrm{~nm},-0.5 \mathrm{~V}, 30 \mathrm{pA})$, (b) STM image of naphthalene molecules dosed at room temperature and annealed to $360 \mathrm{~K}$, imaged at $4 \mathrm{~K}(25$ $\times 25 \mathrm{~nm},-0.5 \mathrm{~V}, 30 \mathrm{pA}$ ), and (c) normalized height histogram of the STM images shown in red (a) and black (b). 
dehydrogenation, the naphthalene molecules are slightly higher and have a broader height variation, indicating that the molecules have started tilting with respect to the surface.

\section{DFT calculations}

DFT calculations were performed to obtain the dehydrogenation reaction pathway energetics and vibrational frequencies to support the interpretation of the SFG results. We explored a large number of possible reaction pathways, but here we only show those with calculated barriers that are likely to be relevant for the experimental conditions. Figure 5 shows the energetics of the reaction pathways and optimized structures, as determined using DFT calculations for (a) the naphthalene dehydrogenation, assuming a stepwise abstraction of hydrogen atoms, and (b) the recombination of two surface hydrogen atoms to $\mathrm{H}_{2}$. The first step in the reaction pathway is the barrier-less adsorption of naphthalene at the $\mathrm{Ni}(111)$ surface. In this exoergic process, an energy of $109 \mathrm{kcal} / \mathrm{mol}$ is released. Unfortunately, a lack of literature makes it difficult to find a comparison for this value; we can, however, compare to the adsorption energy of benzene on $\mathrm{Ni}(111)$. In a recent calorimetry study by Carey et al., ${ }^{55}$ the energetics of adsorption of benzene on $\mathrm{Ni}(111)$ and $\mathrm{Pt}(111)$ was experimentally determined. For benzene on $\mathrm{Ni}(111)$, they found a value of $50 \mathrm{kcal} / \mathrm{mol}$, which is lower than the value we find for naphthalene on $\mathrm{Ni}(111)$. The high coordination of the naphthalene to the surface suggests that the adsorption energy should be higher, and we can assume that the adsorption energy scales linearly with the amount of carbon atoms, similar to cyclic hydrocarbons on $\mathrm{Ru}(001)$ as was shown by Madey and Yates. ${ }^{56}$ Under this assumption, our calculated adsorption energy is not unrealistic.

Upon adsorption, the naphthalene adopts a flat geometry parallel to the surface and undergoes pronounced dearomatization, as previously observed for naphthalene on $\mathrm{Ni}(111)$ and benzene on $\mathrm{Ni}(100) .^{25}$ The sum of $\mathrm{C}_{10} \mathrm{H}_{8}-\mathrm{Ni}$ bond orders (BO), according to DDEC $^{57,58}$ charge and bond analysis, is 5.89 , and the sum of $\mathrm{BO}$ in the $\mathrm{C}$ ring is 13.82 , significantly less than the $\mathrm{BO}$ of the carbon rings in gas phase naphthalene which is 17.05 .

Naphthalene has two inequivalent sets of hydrogen atoms, $\alpha$ and $\beta$, as depicted in the top left corner of Fig. 5, where $\alpha$ and $\beta$ are the four hydrogens adjacent to the carbon atoms shared by both rings and the four hydrogens on the outer carbon atoms, respectively. Dehydrogenation can occur by abstraction of either of these two types of hydrogen atoms. The endoergic detachment of one $\alpha-\mathrm{H}$ atom has a barrier of $23 \mathrm{kcal} / \mathrm{mol}$, and the total energy change with respect to the adsorbed naphthalene structure is $13 \mathrm{kcal} / \mathrm{mol}$, as shown in Fig. 5. Abstraction of a second $\mathrm{a}-\mathrm{H}$ has a barrier of $26 \mathrm{kcal} / \mathrm{mol}$. The total energy change for removal of two $\alpha$ $\mathrm{H}$ is $25 \mathrm{kcal} / \mathrm{mol}$. In the case of $\beta-\mathrm{H}$, the first dehydrogenation step from the adsorbed naphthalene has a barrier of $30 \mathrm{kcal} / \mathrm{mol}$, $7 \mathrm{kcal} / \mathrm{mol}$ higher than the first $\alpha-\mathrm{H}$ abstraction. It is therefore more likely that the first hydrogen abstraction will occur from an a-position.

After the abstraction of two hydrogens from $\alpha$-positions, the naphthalene fragment left behind $\left(\mathrm{C}_{10} \mathrm{H}_{6}-\mathrm{Ni}\right)$ has a total $\mathrm{BO}$ of 7.33, and the sum of the bond orders of the two hydrogen atoms with the $\mathrm{Ni}$ surface is 2.18. This increase in $\mathrm{BO}$, compared to $\mathrm{C}_{10} \mathrm{H}_{8}-\mathrm{Ni}$ (BO 5.89), is due to stronger $\mathrm{C}-\mathrm{Ni}$ coordination by the dehydrogenated carbons. The strong interaction of the naphthalene electronic structure with the Ni substrate results in a pronounced shift of the $\mathrm{Ni}$ atoms and tilting $\left(\sim 7^{\circ}\right)$ of the naphthalene fragment, as illustrated in Fig. 6.

Panel (B) in Fig. 5 shows the reaction pathway for the recombination of two hydrogen atoms, adsorbed on adjacent $\mathrm{Ni}$ atoms to form an $\mathrm{H}_{2}$ molecule and subsequent desorption; this energy barrier is $27 \mathrm{kcal} / \mathrm{mol}$, which is approximately the same value found by

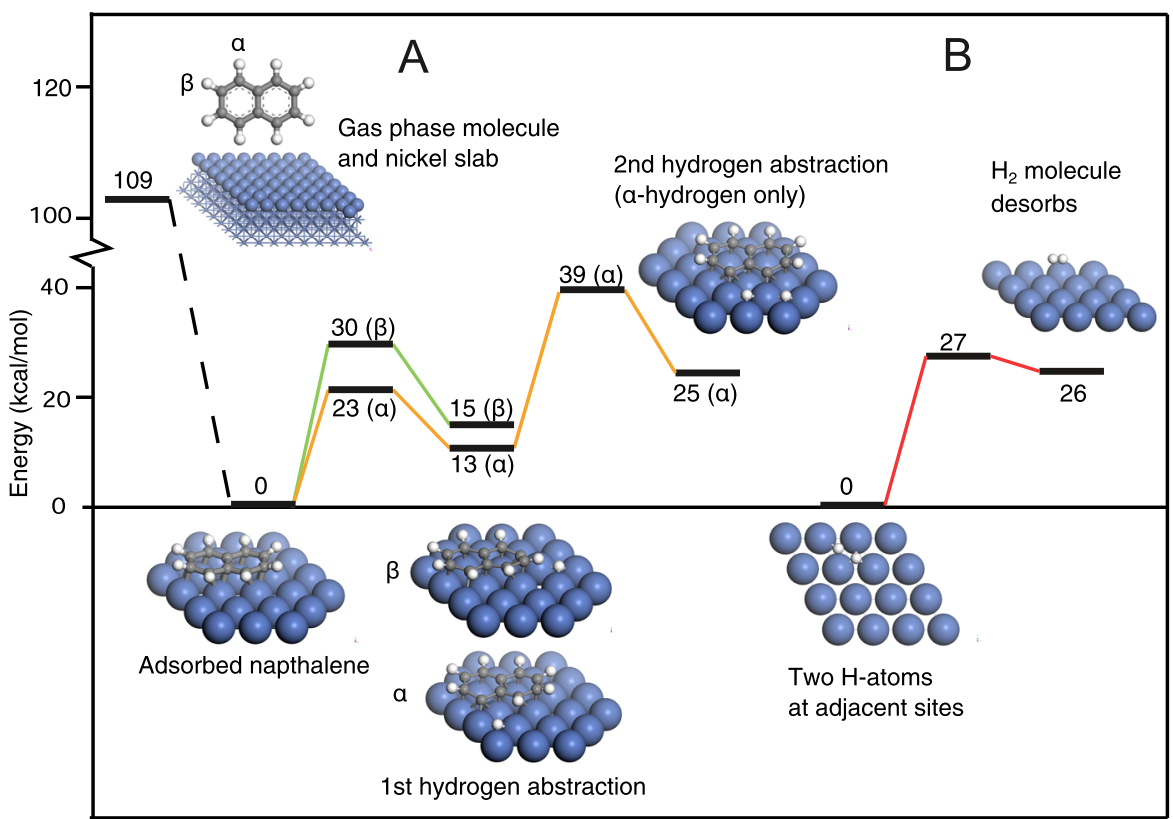

FIG. 5. Energy pathways and optimized structures as determined using DFT calculations for (A) adsorption and dehydrogenation of naphthalene and (B) hydrogen recombination. The energies are presented in $\mathrm{kcal} / \mathrm{mol}$ and displayed relative to the energy of $(A)$ the adsorbed naphthalene molecule and (B) the adsorbed hydrogen atom 


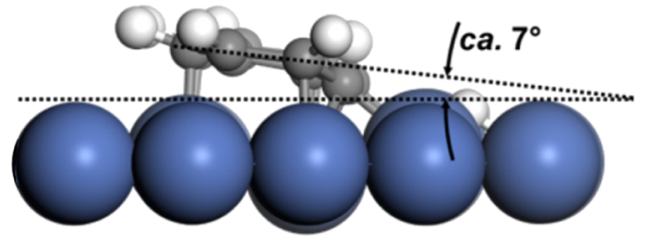

FIG. 6. Side view of the $\mathrm{C}_{10} \mathrm{H}_{6}$ radical resulting from a double hydrogen abstraction from naphthalene. The carbon atoms that lost a hydrogen atom bind stronger to the nickel surface which results in tilting of the molecules with an angle of $\sim 7^{\circ}$.

Kresse $^{59}$ who used DFT to study the potential energy surface of the $\mathrm{H}_{2}$ dissociation process nickel surfaces. The energy barrier for the process in the opposite direction, i.e., the dissociative adsorption of $\mathrm{H}_{2}$ molecule, is as low as $1 \mathrm{kcal} / \mathrm{mol}$.

The energy of the hydrogen atoms at adjacent sites is calculated to be $3-5 \mathrm{kcal} / \mathrm{mol}$ higher than the energy of the two $\mathrm{H}$ atoms at distant sites. In both cases, the $\mathrm{H}$ atom occupies the site hollow sites on the $\mathrm{Ni}(111)$ surface. This energy difference is similar to the energy barrier for diffusion for $\mathrm{H}$ atoms on $\mathrm{Ni}(111)$ as computed by Watson et al. ${ }^{60}$ and experimentally determined by Cao et $a l^{61}$ who found values of $3 \mathrm{kcal} / \mathrm{mol}$ and $4.5 \mathrm{kcal} / \mathrm{mol}$, respectively.

The energy barrier for migration of an $\mathrm{H}$ atom from a position on the surface into the bulk, between the topmost and the second layer of $\mathrm{Ni}$ atoms, was calculated to be $31 \mathrm{kcal} / \mathrm{mol}$. This migration is therefore less likely compared to $\mathrm{H}$-atom recombination and subsequent desorption of the hydrogen molecule.

In order to make comparisons between theory and the SFG experiments, we have calculated vibrational frequencies for the lowenergy species. Harmonic frequencies calculated with DFT are typically too high, and the agreement can often be improved by using a uniform scaling factor. We have determined this factor by comparing the calculated $\left(3125 \mathrm{~cm}^{-1}\right)$ and experimentally measured $\left(3057 \mathrm{~cm}^{-1}\right)$ frequencies for the symmetric $\mathrm{C}-\mathrm{H}$ stretching mode, yielding a value of 0.98 . All of the calculated frequencies we report are scaled by this factor. The magnitude of the scaling factor is consistent with the earlier work by Öberg et al., ${ }^{62}$ where the authors compared calculated and measured SFG frequencies for acetylene on copper.

For the adsorbed naphthalene molecule, we calculate a $\mathrm{C}-\mathrm{H}$ stretching frequency of $2991 \mathrm{~cm}^{-1}$, consistent with the region where we observe an SFG signal for monolayer (chemisorbed) naphthalene $\left(2985-3003 \mathrm{~cm}^{-1}\right)$, resulting from the different preparation methods shown in Fig. 1. For the structure with two abstracted a hydrogens, we calculate a value of $3022 \mathrm{~cm}^{-1}$, in good agreement with the $3029 \mathrm{~cm}^{-1}$ resonance measured after dosing at $480 \mathrm{~K}$. It is lower than the vibrational frequency of the resonance we observed after low temperature dosing followed by flashing to $480 \mathrm{~K}\left(3057 \mathrm{~cm}^{-1}\right)$, which we attribute to the tilted dehydrogenated molecule. We have performed test calculations for structures with the naphthalene rings perpendicular to the surface, which yielded frequencies of $3058 \mathrm{~cm}^{-1}$, and demonstrate the sensitivity of the vibrational spectrum to the geometry of the molecule on the surface. This is consistent with the experimental observation that the preparation temperature influences the structures formed on the surface. The DFT results are in agreement with the results of the STM and SFG experiments and support the interpretation that the naphthalene molecules tilt away from the surface following partial dehydrogenation.

\section{CONCLUSIONS}

The present investigation uses an integrated experimental and theoretical approach to better understand the reaction pathway and surface species present during the dehydrogenation of naphthalene on $\mathrm{Ni}(111)$. The results obtained provide a molecular background for the catalytic steam reforming of tar. We show that naphthalene adsorbs flat on the surface and undergoes significant dearomatization upon adsorption. The dehydrogenation starts at $360 \mathrm{~K}$, and the calculated energy barriers for dehydrogenation are $23 \mathrm{kcal} / \mathrm{mol}$ and $30 \mathrm{kcal} / \mathrm{mol}$ for $\alpha$ and $\beta$ hydrogen, respectively. These calculated barriers for dehydrogenation are comparable to the energy barrier for the formation of the tilted species observed in our SFG measurements at $3057 \mathrm{~cm}^{-1}$. The tilted species is a product of initial dehydrogenation as the dehydrogenated carbon atoms bind stronger to the nickel surface atoms. As a result, the other carbon atoms within the molecule increase in $\mathrm{sp}^{2}$ characteristics and the molecule retrieves some of the aromaticity that was lost upon adsorption. Further heating continues the dehydrogenation, leading to decomposition of the tilted species, the formation and desorption of $\mathrm{H}_{2}$, and the formation of a mixture of carbidic and graphitic surface carbon.

\section{ACKNOWLEDGMENTS}

The project was funded by VR (Dnr: 2015-05242) and SSF. The work was partly carried out within the Swedish Gasification Center (SFC) consortium with funding from the Swedish Energy Agency (Grant No. 34721-2), academic and industrial partners. We thank HZB for the allocation of synchrotron radiation beamtime at the PM4 beamline. We acknowledge the Paul Scherrer Institut, Villigen, Switzerland, for provision of synchrotron radiation beamtime at PEARL beamline of the SLS.

D.J.H. acknowledges financial support from the Swedish Foundation for Strategic Research.

A.L. acknowledges support from the Swedish Research Council (Grant No. 2014-6463) and Marie Sklodowska Curie Actions (Cofund, Project No. INCA 600398).

Y.S. acknowledges the support from the Swedish Research Council (VR) through a Starting Grant (Dnr. 2017-05078).

\section{REFERENCES}

${ }^{1}$ K. Engvall, H. Kusar, K. Sjöström, and L. J. Pettersson, Top. Catal. 54, 949 (2011).

${ }^{2}$ C. Okkerse and H. van Bekkum, Green Chem. 1, 107 (1999)

${ }^{3}$ R. J. Evans and T. A. Milne, in Developments in Thermochemical Biomass Conversion, edited by A. V. Bridgewater and D. G. B. Boocock (Blackie Academic \& Professional, London, 1997), Vol. 2, p. 803.

${ }^{4}$ J. Rostrup-Nielsen and L. J. Christiansen, Concepts in Syngas Manufacture (World Scientific Publishing Company, Singapore, USA, 2011).

${ }^{\mathbf{5}}$ L. Devi, K. J. Ptasinski, and F. J. J. G. Janssen, Biomass Bioenergy 24, 125 (2003).

${ }^{6}$ H. Öström, L. Triguero, M. Nyberg, H. Ogasawara, L. G. Pettersson, and A. Nilsson, Phys. Rev. Lett. 91, 046102 (2003). 
${ }^{7}$ M. G. Yazdi, P. H. Moud, K. Marks, W. Piskorz, H. Öström, T. Hansson, A. Kotarba, K. Engvall, and M. Göthelid, J. Phys. Chem. C 121, 22199 (2017).

${ }^{8}$ D. Dahlgren and J. C. Hemminger, Surf. Sci. 114, 459 (1982).

${ }^{9}$ D. Dahlgren and J. C. Hemminger, Surf. Sci. 109, L513 (1981).

${ }^{10}$ J. L. Gland and G. A. Somorjai, Surf. Sci. 38, 157 (1973).

${ }^{11}$ A. Gavezzotti and M. Simonetta, Surf. Sci. 116, L207 (1982).

${ }^{12}$ V. M. Hallmark, S. Chiang, and C. Wöll, J. Vac. Sci. Technol., B 9, 1111 (1991).

${ }^{13}$ V. M. Hallmark, S. Chiang, J. K. Brown, and C. Wöll, Phys. Rev. Lett. 66, 48 (1991).

${ }^{14}$ G. Santarossa, M. Iannuzzi, A. Vargas, and A. Baiker, ChemPhysChem 9, 401 (2008).

${ }^{15}$ R. Forker, J. Peuker, M. Meissner, F. Sojka, T. Ueba, T. Yamada, H. S. Kato, T. Munakata, and T. Fritz, Langmuir 30, 14163 (2014).

${ }^{16}$ H. Wang, G. Dutton, and X.-Y. Zhu, J. Phys. Chem. B 104, 10332 (2000).

${ }^{17}$ W. Zhao, W. Wei, and J. M. White, Surf. Sci. 547, 374 (2003).

${ }^{18}$ T. Yamada, M. Shibuta, Y. Ami, Y. Takano, A. Nonaka, K. Miyakubo, and T. Munakata, J. Phys. Chem. C 11413334 (2010).

${ }^{19}$ R. F. Lin, R. J. Koestner, M. A. Van Hove, and G. A. Somorjai, Surf. Sci. 134, 161 (1983).

${ }^{20}$ T. Rockey and H.-L. Dai, Surf. Sci. 601, 2307 (2007).

${ }^{21}$ K. H. Frank, P. Yannoulis, R. Dudde, and E. E. Koch, J. Chem. Phys. 89, 7569 (1988).

${ }^{22}$ L. E. Firment and G. A. Somorjai, J. Chem. Phys. 63, 1037 (1975).

${ }^{23}$ W. X. Huang and J. M. White, J. Phys. Chem. B 108, 5060 (2004).

${ }^{24}$ E. L. Kolsbjerg, G. Goubert, P. H. McBreen, and B. Hammer, J. Chem. Phys. 148, 124703 (2018).

${ }^{25} \mathrm{M}$. Weinelt, N. Wassdahl, T. Wiell, O. Karis, J. Hasselström, P. Bennich, and A. Nilsson, Phys. Rev. B 58, 7351 (1998).

${ }^{26}$ Y. Lu and X. Yang, Carbon 81, 564 (2015).

${ }^{27}$ Z. Li, P. Wu, C. Wang, X. Fan, W. Zhang, X. Zhai, C. Zeng, Z. Li, J. Yang, and J. Hou, ACS Nano 5, 3385 (2011).

${ }^{28}$ W. Zhao, S. M. Kozlov, O. Höfert, K. Gotterbarm, M. P. A. Lorenz, F. Viñes, C. Papp, A. Görling, and H.-P. Steinrück, J. Phys. Chem. Lett. 2, 759 (2011).

${ }^{29}$ B. Wang, M. König, C. J. Bromley, B. Yoon, M.-J. Treanor, J. A. Garrido Torres, M. Caffio, F. Grillo, H. Früchtl, N. V. Richardson, F. Esch, U. Heiz, U. Landman, and R. Schaub, J. Phys. Chem. C 121, 9413 (2017).

${ }^{30}$ J. Lahiri, T. Miller, L. Adamska, I. I. Oleynik, and M. Batzill, Nano Lett. 11, 518 (2011).

${ }^{31}$ R. Rameshan, V. Vonk, D. Franz, J. Drnec, S. Penner, A. Garhofer, F. Mittendorfer, A. Stierle, and B. Klotzer, Sci. Rep. 8, 2662 (2018).
${ }^{32}$ L. L. Patera, C. Africh, R. S. Weatherup, R. Blume, S. Bhardwaj, C. CastellarinCudia, A. Knop-Gericker, R. Schloegl, G. Comelli, S. GHofmann, and C. Cepek, ACS Nano 7, 7901 (2013)

${ }^{33}$ M. Bonn, H. Ueba, and M. Wolf, J. Phys.: Condens. Matter 17, S201 (2005).

${ }^{34}$ E. Giangrisostomi, R. Ovsyannikov, F. Sorgenfrei, T. Zhang, A. Lindblad, Y. Sassa, U. B. Cappel, T. Leitner, R. Mitzner, S. Svensson, N. Mårtensson, and A. Föhlisch, J. Electron Spectrosc. Relat. Phenom. 224, 68 (2018).

${ }^{35}$ S. Grimme, J. Comput. Chem. 27, 1787 (2006).

${ }^{36}$ G. Kresse and J. Hafner, Phys. Rev. B 47, 558 (1993).

${ }^{37}$ G. Kresse and J. Hafner, Phys. Rev. B 49, 14251 (1994).

${ }^{38}$ F. Birch, Phys. Rev. 71, 809 (1947).

${ }^{39}$ M. Methfessel and A. T. Paxton, Phys. Rev. B 40, 3616 (1989).

${ }^{40}$ T. A. Manz and D. S. Sholl, J. Chem. Theory Comput. 6, 2455 (2010).

${ }^{41}$ T. A. Manz and D. S. Sholl, J. Chem. Theory Comput. 8, 2844 (2012).

${ }^{42}$ C. Kittel, Introduction to Solid State Physics, 8th ed. (John Wiley \& Sons, Inc, 2005), p. 328.

${ }^{43}$ G. Henkelman and H. Jonsson, J. Chem. Phys. 113, 9978 (2000).

${ }^{44} \mathrm{H}$. Arnolds and M. Bonn, Surf. Sci. Rep. 65, 45 (2010).

${ }^{45}$ H. A. Pearce and N. Sheppard, Surf. Sci. 59, 205 (1976).

${ }^{46}$ P. Jakob and D. Menzel, Surf. Sci. 220, 70 (1989).

${ }^{47}$ S. Lehwald, H. Ibach, and J. E. Demuth, Surf. Sci. 78, 577 (1978).

${ }^{48}$ H. Öström, A. Föhlisch, M. Nyberg, M. Weinelt, C. Heske, L. G. M. Pettersson, and A. Nilsson, Surf. Sci. 559, 85 (2004).

${ }^{49}$ F. S. Thomas, N. S. Chen, L. P. Ford, and R. I. Masel, Surf. Sci. 486, 1 (2001).

${ }^{50}$ L. Asplund, U. Gelius, S. Hedman, K. Helenelund, K. Siegbahn, and P. E. M. Siegbahn, J. Phys. B: At. Mol. Phys. 18, 1569 (1985).

${ }^{51}$ U. Gelius, S. Svensson, H. Siegbahn, E. Basilier, Å. Faxälv, and K. Siegbahn, Chem. Phys. Lett. 28, 1 (1974).

${ }^{52}$ R. Haerle, E. Riedo, A. Pasquarello, and A. Baldereschi, Phys. Rev. B 65, 045101 (2001).

${ }^{53}$ A. Wiltner and C. Linsmeier, Surf. Sci. 602, 3623 (2008).

${ }^{54}$ E. O. F. Zdansky, A. Nilsson, and N. Mårtensson, Surf. Sci. 310, L583 (1994).

${ }^{55}$ S. J. Carey, W. Zhao, and C. T. Campbell, Surf. Sci. 676, 9 (2018).

${ }^{56}$ T. E. Madey and J. T. Yates, Jr., Surf. Sci. 76, 397 (1978).

${ }^{57}$ N. G. Limas and T. A. Manz, RSC Adv. 8, 2678 (2018).

${ }^{58}$ T. A. Manz, RSC Adv. 7, 45552 (2017).

${ }^{59}$ G. Kresse, Phys. Rev. B 62, 8295 (2000).

${ }^{60}$ G. W. Watson, R. P. K. Wells, D. J. Willock, and G. J. Hutching, J. Phys. Chem. B 105, 4889 (2001).

${ }^{61}$ G. X. Cao, E. Nabighian, and X. D. Zhu, Phys. Rev. Lett. 79, 3696 (1997).

${ }^{62}$ H. Öberg, Y. Nestsiarenka, A. Matsuda, J. Gladh, T. Hansson, L. G. M. Pettersson, and H. Öström, J. Phys. Chem. C 116, 9550 (2012). 